\title{
AN INNOVATIVE BRACE WITH PNEUMATIC THRUSTS FOR SCOLIOSIS TREATMENT
}

\author{
M.G. ANTONELLI ${ }^{1}$, P. BEOMONTE ZOBEL ${ }^{1}$, P. RAIMONDI ${ }^{1}$, T. RAPARELLI ${ }^{2} \&$ G. COSTANZO $^{3}$ \\ ${ }^{1}$ Department of Mechanical Engineering, Energy and Management, University of L'Aquila, Italy. \\ ${ }^{2}$ Department of Mechanics, Politecnico di Torino, Italy. \\ ${ }^{3}$ Department of Orthopaedic Surgery, University of Rome "La Sapienza" Polo Pontino, Italy.
}

\begin{abstract}
Idiopathic scoliosis is the most common spinal deformity, and the brace treatment is the fundamental therapy. Brace works on the curve via the pressure they exert on the rib cage, usually on three points, to push against the progressive abnormal curvature of the spine and to reduce the local spasticity. Considering the possibility of the spine collapse, due to the six degrees of freedom of each vertebras with respect to the adjacent one, spine needs more thrusts relating to the curvature extension. This paper is focused on an innovative brace working by a three-dimensional thrusts system. The brace is internally covered by air pocket devices. Design and prototype of air pocket device is described. Air pockets apply the necessary corrective thrusts, at many levels of the spine, by compressed air at the specific pressure required in that area of the rib cage. Thrusts can be modified and checked. The design of the brace is presented together with a basic prototype, the design and the prototype of the air pocket device. First experimental tests on a simplified prototype of brace are also described. Finally, a pre-clinical test is performed by a healthy volunteer to validate the new brace concept.
\end{abstract}

Keywords: brace design, FEM modelling, pneumatic pad, scoliosis brace.

\section{INTRODUCTION}

Scoliosis is a spatial abnormal curvature of the spine: a lateral curvature on the frontal plane, a modification of the curves in the sagittal plane and a vertebral rotation in the horizontal plane [1]. The most common form is the idiopathic scoliosis. The incidence of it in the general population ranges from $0.2 \%$ to $0.3 \%$, depending on the size of the curve, with a prevalence of $2-3 \%$ in schoolaged children [2]. More is known about the natural history of curve progression in adolescent idiopathic scoliosis, which is dependent on the patient's skeletal maturity, the curve pattern and the curve magnitude [3]. On the contrary, the pathogenesis of it is still obscure. There are tentative aetiologies for idiopathic scoliosis, including, for example, differences in the growth rate of the anterior and posterior part of the spine. Recent evidences show that the disease has hereditary basis, supported by clinical manifestations as well as family studies that revealed the familiar tendency of idiopathic scoliosis [2]. The study of scoliosis has ancient origins. The deformity was firstly described by Ippocrate, around $400 \mathrm{BC}$; the diagnosis, the causes and, above all, the treatments have been the focus of a great deal of research. Ippocrate tried to reduce the scoliosis curvature using a table, to which the patient was tied, and a person who hopped on him pushing the spine. In the following centuries many scientists (Galeno, Parrè, Delpech and others) were engaged in finding a therapeutic method for scoliosis: the application of a mechanical pushing to convex parts of the spine, the extension of the spine by a traction force or by the weight of the patient. But only in the 1840 starts the activity of orthopaedic medical centres in France specifically devoted to the correction of the scoliosis. In the following decades the scientists move from the traction fixed devices to different ideas of brace. The brace of Shanz-Milwaukee (1945) and the optimised version of Blount and Schmidt (1958) are the most significant ones of that period. Towards the end of the 20th century there was a growing interest in research activities on scoliosis and on the improvement of braces. The brace is used to prevent and to slow the curve progression, but the results are difficult to predict. Braces work on the curve via the pressure they exert on the rib cage, usually on three points, to push against the 
progressive abnormal curvature of the spine and to reduce the local spasticity. The brace applies direct thrusts, as primary correction effect, and reaction thrusts, at the contact area between brace and thorax.

In Fig. 1, three different curvature of the scoliotic spine are shown. The figure depicts the principal thrust, black arrow, and the reaction thrusts, the white ones, that the brace applies to the spine. The reaction thrusts apply in the pelvic shell and in the top part of the brace. The principal and the reaction thrusts don't succeed to apply the load to whole of the spine but just on the apex of the curvature and on the pelvic and on the neck regions. To increase the surface of the rib cage where the thrusts are applied, so that the correction pushing is applied to a greater part of the spine, some pads are used.

Brace treatment for idiopathic scoliosis has good support in published studies of Negrini et al. [1] and Labelle et al. [4]. It is non-invasive and preserves growth, motion and function of the spine [3]. Braces can be divided in two categories: fixed and removable. The choice of the category depends on the age, on the spine conditions and on the prognosis of scoliosis. The latter one is based on some clinical data (hump, Cobb angle, torsional angle, age, etc.) and on its localisation and curve pattern. After this step, the clinician specialised in spinal diseases, on the basis of his experience, will choose the specific brace, if necessary and useful.

For idiopathic scoliosis, removable brace treatment is a fundamental therapy, on condition that brace is light, patient compliance, constructed by an orthotist specialised in the construction of the prescribed brace system. The use of removable braces is recommended in scoliosis curvatures with the Cobb angle between $20 \pm 5^{\circ}$ and $40 \pm 5^{\circ}$ [1] depending on the authors. In this case the therapy is most effective with a physical rehabilitation. The therapeutic rehabilitation is addressed for Cobb angle less than $15 \pm 5^{\circ}$, whereas the fixed brace and the surgical treatment is the therapy for the Cobb angle greater than $40 \pm 5^{\circ}$.

Many removable brace systems have been proposed and developed in different countries and many different types are on the market, often specialised for a specific curvature and for a specific correction of the spine. The Lyon brace, designed in France, is indicated for lumbar and for thoracolumbar curves; the Cheneau brace, also designed in France, has three different versions: for single lumbar curves, for lumbar curves and for thoracic curve with an apex at L4/T10; La Padula brace, designed in Italy, is indicated for the lower lumbar and thoracolumbar curves; the Boston brace,

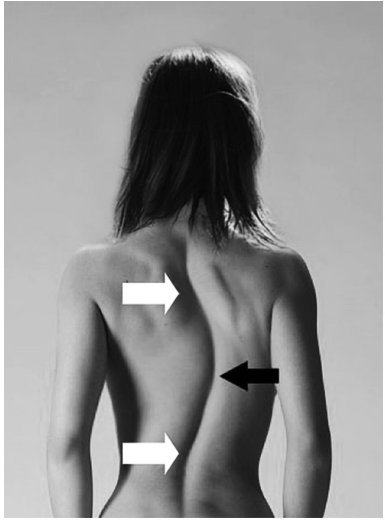

(a)

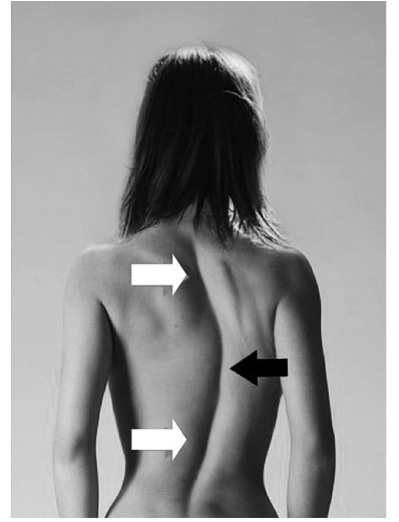

(b)

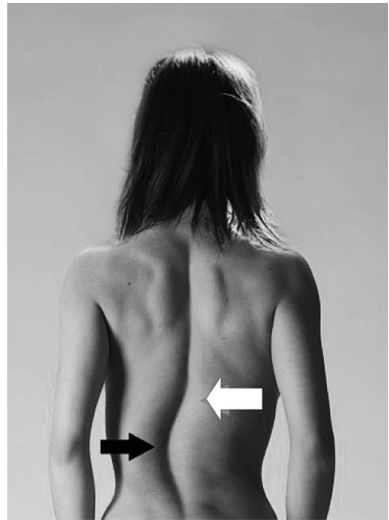

(c)

Figure 1: Example of thrusts application as some curvatures of the scoliotic spine: (a) thoracic, (b) thoracolumbar, and (c) lumbar. 
designed in USA, is indicated for thoracolumbar curves. The Milwaukee brace, also designed in USA, is the most common one for the scoliosis treatment. It is used for thoracolumbar curves and for double curves. This brace uses two distinct principles: the three points thrust and the extension of the spine. The brace is linked to the pelvic region and has a top part to constraint the chin. The patient has to stay with the chin far from the constraint, and so a traction force is applied to the spine. To obtain this traction force, the Milwaukee brace has a pelvic shell modelled to reduce the lumbar lordosis. A lateral shell applies the main thrust.

This paper is focused on a new brace proposed for the scoliosis treatment. The presented brace is a suitable adapted Cheneau brace that relies on a three-dimensional thrusts system realised by air pocket devices, fixed inside it, to create an actuating shell around the entire rib cage. In each air pocket the air is inflated to a specific pressure value in order to apply the necessary corrective thrust in that specific area of the rib cage. The thrust can be modified and checked. In this way it is possible to apply different values of thrust at many levels of the spine so that the action on it can be more effective. The idea of the brace is presented together with a basic prototype, the design and the prototype of the air pocket device. Due to the behaviour of the material of the air pockets, a numerical model, implemented by a finite element code, was developed as a useful design tool for air pockets. The numerical model and its experimental validation are presented. First experimental tests on a simplified prototype of brace were performed. Finally, a pre-clinical test was performed by a healthy volunteer to validate the new brace concept.

\section{NEW BRACE CONCEPT}

The proposed brace is based on the 'global thrust', that is a three-dimensional thrusts system acting on the entire rib cage. The idea is to have thrusts at many levels of the spine, not only at three levels as with a traditional brace. This idea is supported by the consideration that the spine is formed by vertebras and each of them has six degrees of freedom (dof) with respect to the adjacent one. These six dof have a limited movement range, but it exist the possibility of a collapse of the spine, where it has the maximum value of the instability, when the patient wears a brace with three thrusts, as shown in Fig. 2. It could happen because the spine is constrained only in correspondence of the thrusts point and in the lower and upper ends. The remaining unconstrained parts of the spine, due to the vertebra dof, can be subdued to further uncontrolled curvature.

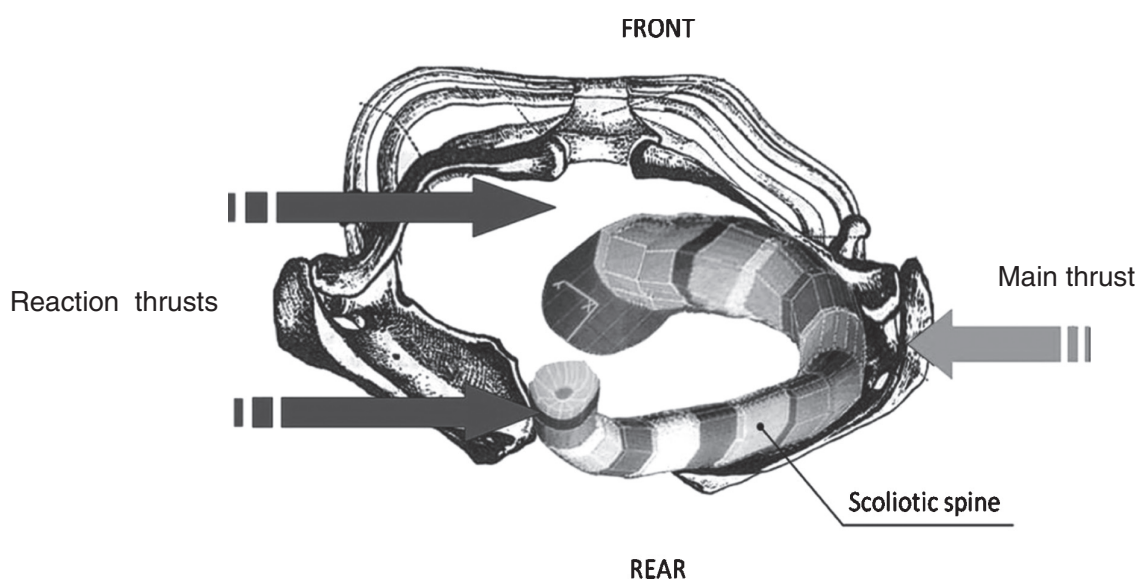

Figure 2: Scoliotic spine with main and reaction thrusts in a top view. 
From the point of view of the authors, the spine can be considered as made by 24 cylindrical bodies: the vertebras. The ends of each body articulate by pads of elastic or cartilaginous tissue with those of adjacent ones. This cylinder of 24 segments with a specific configuration, depending on the shape of the vertebras, is linked to the rear part of the thorax, from the internal side, with a rotational joint, so that the spine can swing relative to the thorax, as represented in Fig. 3a. For this reason, the helical shape of the scoliotic spine has to be constrained inside a column of thrusts surrounding the entire spine. It means that the spine needs more thrusts relating to the extension of the curvature, as shown in Fig. $3 \mathrm{~b}$ and c. This principle is as more true as more the extension of the curvature involves a high number of vertebras: a scoliotic curvature extended for 10 vertebras requires more thrusts than a curvature extended for only 5 vertebras.

To provide the displacement, i.e. translation and rotation, of the spine in order to correct its curvature, the braces have to apply the thrusts on the rib cage. The greater is the surface where the thrust is applied the lesser is the possibility that the spine moves in unwanted directions. In the same time it has to be possible to control the intensity, the direction and the contact area of the thrusts. It is also important to consider that the intensity of the thrust has to be modified during the treatment, according to the modification of the spine curvature.

To satisfy the above mentioned requirements, the structural part of the new brace, the frame, could be as a traditional brace, except for the internal cover that is made by a shell of air pocket devices in compliant material, fixed on it and in contact with the whole rib cage. Each device is tuned individually, in order to obtain the wanted value of thrust, and each thrust has the possibility to be modified and checked.

The application of the brace should be as follows. At first the value of thrusts to be applied to the rib cage for reduction of the scoliotic curvature has to be settled; then the value of pressure necessary for each device, to obtain those thrusts system, has to be calculated for each point of the scoliotic curvature; finally each device has to be tuned at the specific value. In this way many thrusts are applied to the scoliotic curvature of the spine, one for each needing vertebra and composed by more radial components, except for the expanding part of the thorax.

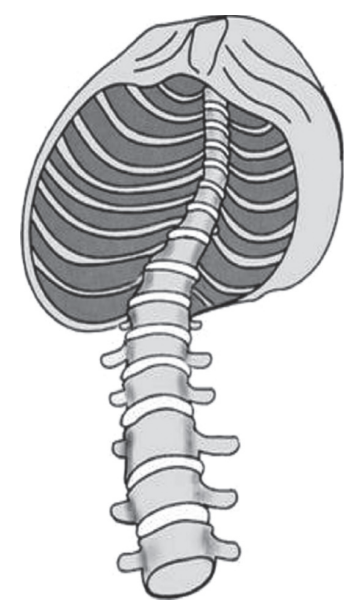

(a)

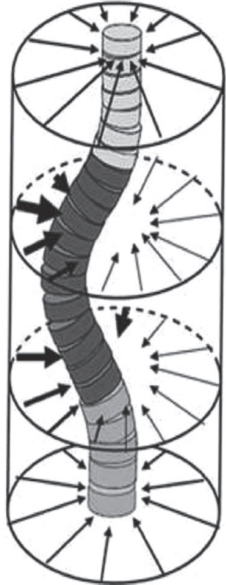

(b)

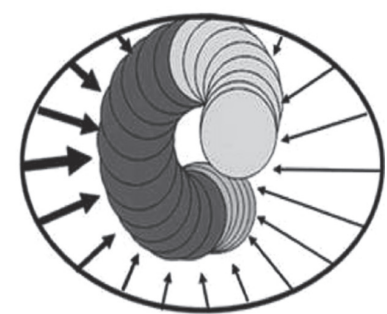

(c)

Figure 3: (a) Axonometric view of the scoliotic spine and the thorax; (b) axonometric view of the scoliotic spine model; (c) top view of the scoliotic spine model. 


\section{MATERIALS AND METHODS}

The main objective of this research is the development of a suitable thrust device to put in practice the new brace concept. The measurement of thrusts in ordinary scoliotic braces is necessary as preliminary data. Based on these results, the technical specifications of the device were defined. Then it was possible to go on with the design and the prototyping. The design requires the development of a numerical model, implemented by a finite element code, also because the silicone and air compressed technology was used to construct the device. Finally the experimental tests were used to measure the performance of the thrust device, named 'air pocket device', as stand alone device and assembled in the new brace.

\subsection{Measurement of typical thrusts values}

The knowledge of thrusts values in a scoliotic brace is necessary to design the air pocket device. For this goal some experimental tests have been scheduled with four scoliotic patients, selected from the clinician, to measure the contact pressure, and then the thrust, between the pad, normally used in Cheneau brace, and the rib cage. To know the thrusts value at the pad interface is useful for the idea of the new brace: air pocket device has to act as a classic pad, with the difference that it can be distributed all around the rib cage. All the patients selected have the same characteristic: a diagnosis of scoliosis and a therapy based on a three points Cheneau brace.

The measurement set up is made by a sensor matrix, a conditioning unit, all manufactured by Medizintechnik Gmbh [5], a data acquisition board NIDAQ 6036E of National Instruments and a Personal Computer. The sensor matrix has a dimension of $80 \times 85 \mathrm{~mm}$ and a thickness of $2.3 \mathrm{~mm}$. It is made by eight couples of pressure and temperature sensors, that are positioned in two rows, and each couple of pressure and temperature sensors are annealed in a gel cell to distribute the pressure on the sensor and to protect against the shock. Each couple of sensors appears as a single sensor and it has a dimension of $11.5 \times 27.5 \mathrm{~mm}$. The maximum value of the pressure that each sensor can measure is $0.2 \mathrm{MPa}$ with a maximum error of $5 \%$ (i.e. $\pm 5 \mathrm{kPa}$ ). Figure $4 \mathrm{a}$ shows the position of the eight sensors in the sensor matrix.

A simple protocol was defined to carry out these experimental tests. The protocol is as follows:

1. the clinician gives to the patient a complete information about the experimental test (goal, procedure, risk) and the patient accepts it signing a consensus agreement;

2. the patient wears the brace in the correct way;

3. the sensor is inserted in a disposable sterile small bag;

4. a data acquisition starts to check the sensor and to read the offset, i.e. the zero error of the instrument, before inserting the sensor;

5. the brace is removed from tension to insert the sensor between the thrust point and the rib cage. The positioning of the sensor is a delicate step because it is necessary to avoid the presence of wrinkles and the sensor has to remain finely in contact with the rib cage and the pad;

6. the brace is tensioned and the first data acquisition starts with the patient in erect position; a check on the acquired data is made, so that if something is wrong the test can be repeated;

7. a second acquisition starts with the patient in seated position; a check on the acquired data is made, so that if something is wrong the test can be repeated;

8. is it the first measurement point? If the answer is yes, go back to point 5 to go on with the second measurement point. If not, remove the brace from tension to recover the sensor: the test with the patient is completed. 
(a)

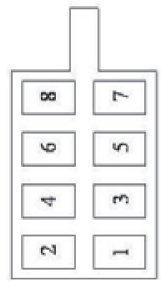

(b)
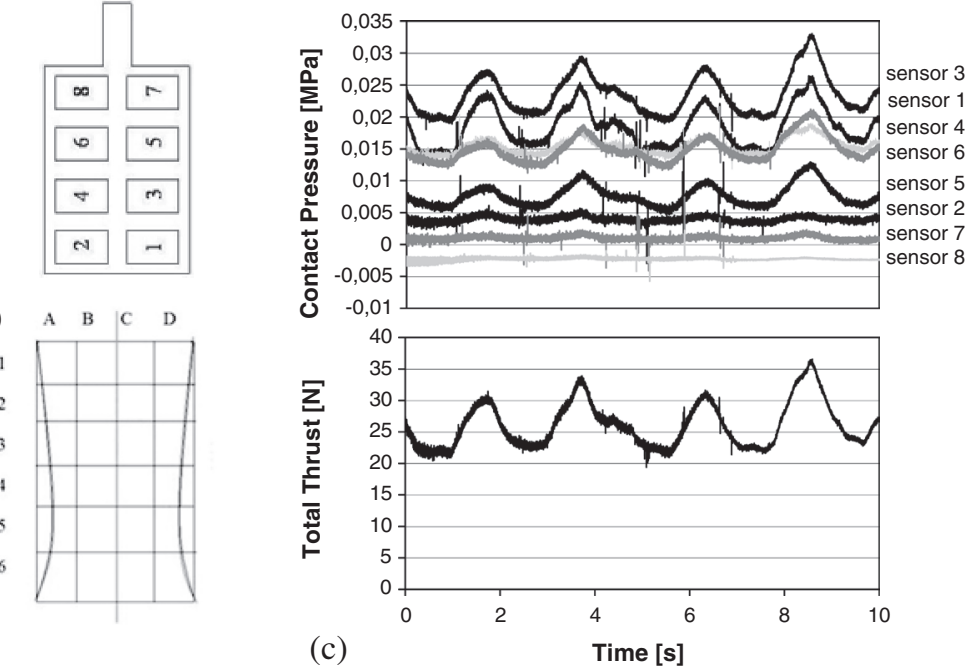

Figure 4: (a) Drawing of sensor matrix with sensors location; (b) grid of the back to locate the measurement points; (c) example of contact pressures measured by sensors and the respective total thrust.

All the signals are acquired at a sample frequency of $1 \mathrm{kHz}$ for an amount of 10,000 acquired samples, i.e. $10 \mathrm{~s}$ as acquisition period. The total thrust $\mathrm{F}$ is calculated by eqn (1):

$$
F=A \cdot \sum_{i=1}^{8} p_{i}
$$

where $p_{i}$ is the pressure measured by the sensor $i$ and $A$ is the area $\left(316 \mathrm{~mm}^{2}=11.5 \times 27.5\right)$ of each sensor. A grid of the back was used to locate the position of the measurement points for each patient, as shown in Fig. 4b. The measurement points of the patients, from 1 to 4, are respectively: $2 \mathrm{C}$ and 4B, 2C and 3B, 3C and 4A, 1B and 1C. In Fig. 4c, it is possible to see an example of the acquired pressure raw data, for each of the eight sensors, between the pad and the rib cage and the respective total thrust.

In Fig. 4c, data are oscillating because of the breathing: the change of the volume of the rib cage modifies the contact pressure and the thrusts. The maximum measured thrust value is $37 \mathrm{~N}$ (patient no. 1 in seated position, point $2 \mathrm{C}$ ). In all the tests the maximum pressure value is measured in seated position. This is due to the kyphotic position of the upper back in many people when seated. Some measurements have shown very low level of pressure, less than 4 N. Probably in this cases a contact-no contact situation occurs at the interface pad rib cage.

\subsection{Air pocket device}

Following the measurement of the thrusts and considering the goal of the brace, the technical specifications of the air pocket device are defined. Specifically the device has:

- to exert a maximum level of force bigger than $40 \mathrm{~N}$, on a area of about $80 \times 80 \mathrm{~mm}^{2}$;

- to be adjustable in order to modify the thrust force during the brace therapy;

- to use a suitable technology to fix the thrust at the desired value during the treatment period; 
- to be provided with a suitable architecture in order to be integrated each other, so that a covering of devices inside the shell is possible, to apply the thrusts where they need;

- to produce a soft contact with the rib cage, for the maximum comfort of the brace.

To satisfy the technical specifications, a thrust device constructed in silicone material that can be inflated by air was conceived. A squared shape was chosen in order to integrate the air pocket devices inside the shell; silicon rubber (Silastic 3481 of Dow Corning) was chosen for its softness and its compatibility with the skin. The air pocket device was thought to be made by a chamber, constructed by two layers of silicon rubber linked each other on the edge, that can be pressurised. A hole has to be used to inlet and outlet the air from the pocket. Pressurising the air inside the pocket, the volume grows as a balloon, so that a thrust is obtained by the contact with the rib cage opposing to the volume of expansion. The air pocket device shows a strongly nonlinear behaviour by which output force depends on both the pressure inside the pocket and its volume. It is due to large strains to which it is subjected and to the constitutive material: silicon rubber, as all elastomeric materials, has a nonlinear $\sigma-\varepsilon$ relationship. For this reason, before designing the air pocket device, it was useful to define a numerical model as a design tool able to predict the behaviour of air pockets. The principal aim was to study the relationship among the main design parameters of the air pocket and its performances: in particular, thrusts exerted by the air pocket as regards pressure values inside the chamber and chamber deformations.

The numerical model was implemented by a finite element model, developed by ANSYS code. It is a three-dimensional and parametric model. To reduce the number of nodes and then the computing time, the finite element model performs a quarter of air pocket, because of its geometry is twice symmetric. For the same reason, the lower layer of the air pocket was modelled with a rigid material, to study only the behaviour of the upper one.

Three different kinds of elements, chosen from ANSYS library [6], were used in the model: SOLID45 elements to model the rigid material inside the lower layer; LINK8 elements used as sensors to measure the exerted thrusts or as constrains of the maximum admitted displacement values; HYPER86 elements to model the silicone rubber. Due to the nonlinear behaviour of the silicone rubber, a nonlinear analysis by Newton-Raphson method was performed. The two coefficients Mooney-Rivlin formulation was applied to HYPER86 elements.

Two different models were implemented: one for the isometric test modelling and one for the isotonic test modelling. In the isometric test, consisting in constant deformation experiments, the force versus pressure inside the chamber is computed; in the isotonic test, consisting in constant force experiments, the displacement of the upper layer versus pressure is computed. In the isometric test modelling, input parameters, besides geometric ones, are pressure value and maximum admitted displacement value; in the isotonic test modelling, pressure value and force acting on the upper layer. Figure 5a and $\mathrm{b}$ show respectively the isotonic test model and the isometric one as an output of the calculation.
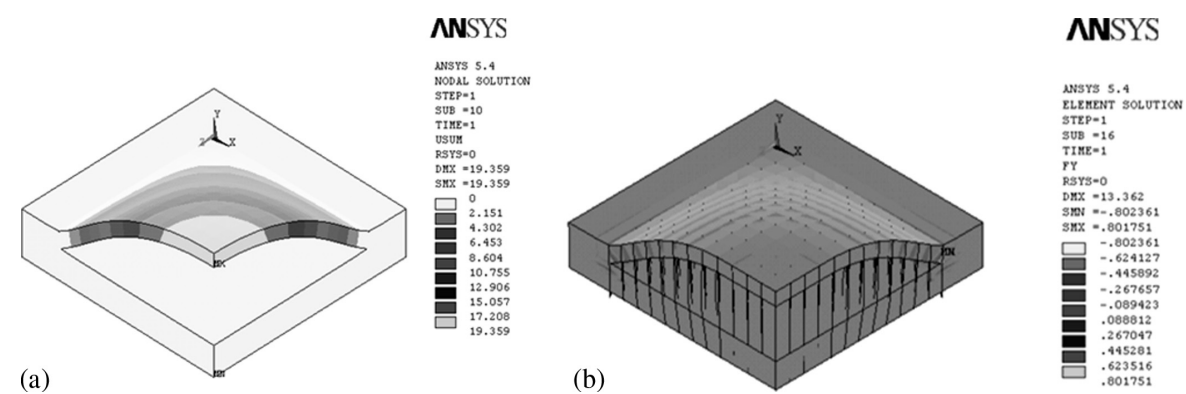

Figure 5: (a) Isotonic test model and (b) isometric test model. 
An experimental prototype of the air pocket device was constructed with the same values of geometrical parameter of the numerical model to carry out an experimental validation.

The geometrical parameters are as follows:

- external edge dimension (squared shape): $120 \mathrm{~mm}$;

- chamber edge dimension (squared shape): $96 \mathrm{~mm}$;

- thickness of the lower layer (rigid material included): $7 \mathrm{~mm}$;

- thickness of the upper layer: $4 \mathrm{~mm}$.

In Fig. 6, the experimental prototype is shown. The manufacturing process was defined and tested and a simple technological set up was developed. The experimental validation of the numerical model was performed by means of isometric and isotonic tests of the experimental prototype. The design and the construction of a simple test bed were necessary to perform these tests. In isometric test, constrains are made by two simple metal plates. The deformation is fixed at different values by a simple screw-nut system that links the two plates. The deformation is measured by a measuring rod and a load cell on the screw is used for the traction force. A manometer measures the air pressure in the air pocket, modified by a pressure regulator, and a portable tensionmeter gives the tension value of the load cell to calculate the traction force. For isotonic test, the prototype is placed on two little plastic blocks. The constant load is obtained by a gravitational mass located on the upper layer of the prototype. In Fig. 7, isometric and isotonic test beds are shown.
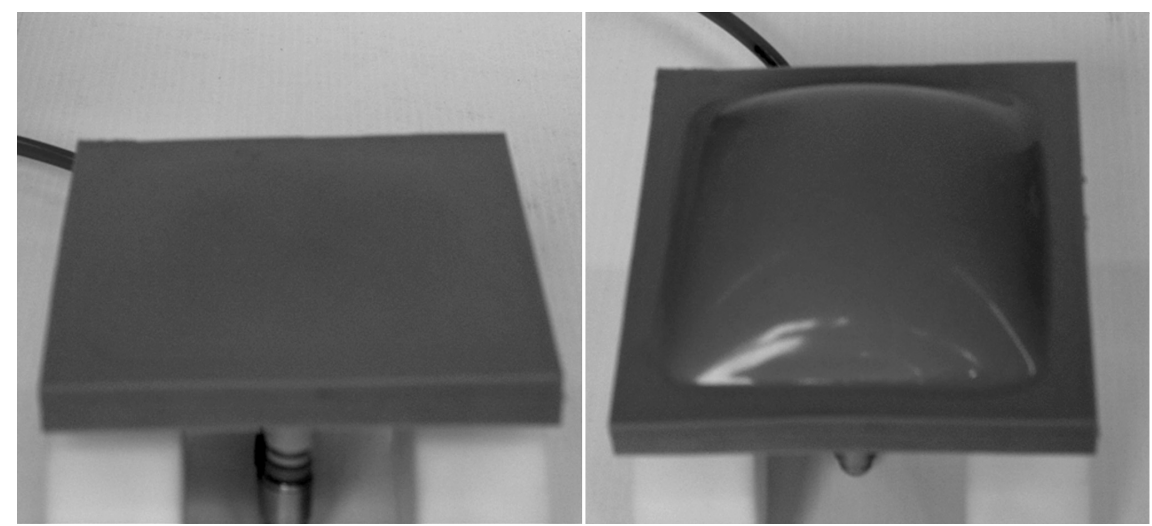

Figure 6: Experimental prototype for numerical model validation.
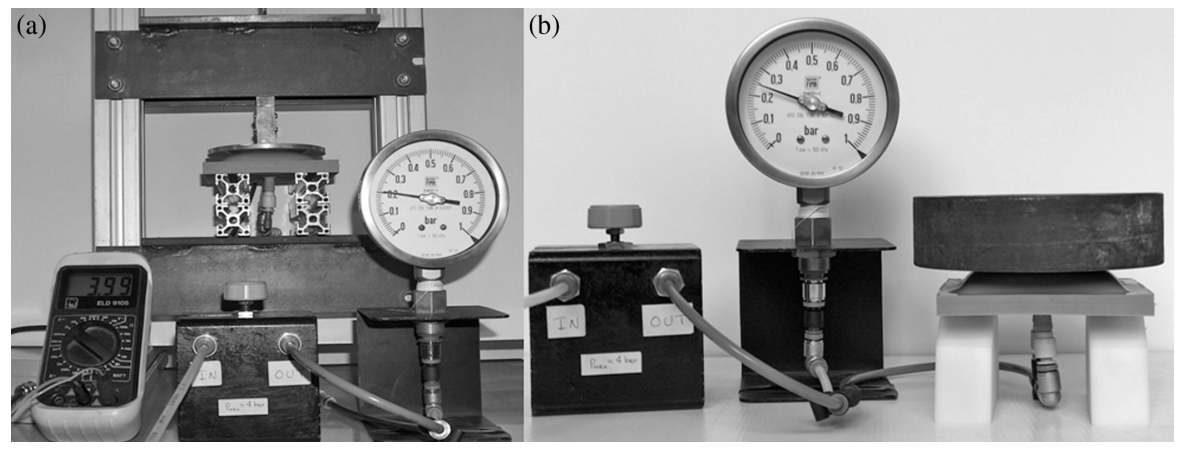

Figure 7: (a) Isometric test bed and (b) isotonic test bed. 
Results obtained from the numerical model and the experimental prototype, in the same test conditions, are strictly correlated. The behaviour of the numerical model is close to the experimental prototype. From the comparison of the obtained results, as shown in Fig. 8, the maximum error registered in isometric test is about $10 \%$ and the maximum error in isotonic test is about $14 \%$. During both these tests, the prototype showed a hysteretic behaviour: two separate curves are obtained increasing the pressure inside the pocket from zero to the target value and then decreasing to zero. This phenomenon is already described in literature for elastomeric materials [7], and the cause of hysteresis seems to be the viscoelastic phenomenon. Considering the limited error margin and the hysteretic behaviour, the numerical model is considered reliable.

Several simulations were performed by the numerical model in order to optimise the design of the air pocket device of the new brace. Finally the best set of geometrical parameters fitting the technical specifications was the following: external edge dimension (squared shape) $=100 \mathrm{~mm}$, chamber edge dimension (squared shape) $=80 \mathrm{~mm}$, thickness of the lower layer $=4 \mathrm{~mm}$, thickness of the upper layer $=4 \mathrm{~mm}$. Ten air pocket devices were manufactured to be used for the brace, using the same technological set up. A leak free valve was used for the inlet and the outlet of the air, so that the chosen value of pressure is used for the brace treatment. The leak free valve is also used to fasten the device to the brace by screwing a nut onto it. New experimental tests were scheduled with these prototypes.

A first set of experimental tests was performed to measure the maximum force applied by the air pocket device and the maximum test pressure. They were measured respectively as $100 \mathrm{~N}$ and $0.065 \mathrm{MPa}$. A second set of experimental tests was performed to check air leaks mainly at the interface between the air pocket and the valve. Two kinds of test were scheduled. In both, air pockets were inflated at $0.05 \mathrm{MPa}$ checking the pressure for $48 \mathrm{~h}$. A first simple test was performed putting the air pocket under water and checking the birth of air bubbles. A second test was performed loading each air pocket with a weight of 98.1 N. No air pocket devices showed air leak. A third set of experimental tests was performed to measure the burst pressure. Two kinds of test were scheduled. In both, air was inflated until burst occurred. A first test was performed at null deformation, constraining each air pocket between two rigid plates. The burst pressure was around $1 \mathrm{MPa}$. A second test was performed in free deformation condition. Some problems arose in doing this test, because of the nut for fixing the valve to the air pocket. When the volume of the air pocket device grows the fixing hole of the valve becomes larger. For this reason the valve was ejected at a pressure value of about $0.055 \mathrm{MPa}$. In the internal side of the brace the maximum deformation at design level is less than $10 \mathrm{~mm}$. At this value the burst pressure is higher than $0.4 \mathrm{MPa}$, so that the maximum operative pressure is settled at $0.065 \mathrm{MPa}$. Finally isometric and isotonic tests were performed in order to obtain the control chart of the air pocket devices. The same previously described test beds were used. The isometric tests were performed by regulating the displacement from 0 to $40 \mathrm{~mm}$.

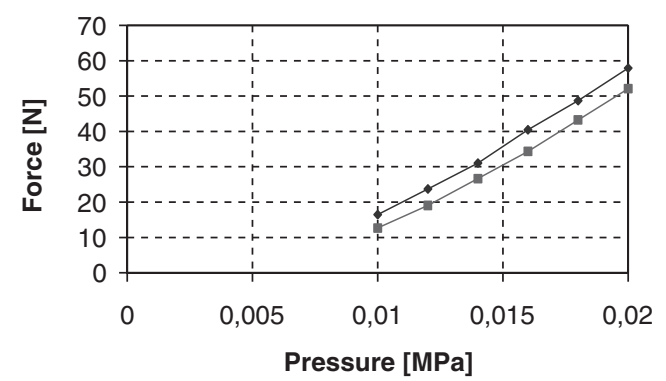

(a) $\quad-$ experimental prototype - - numerical model

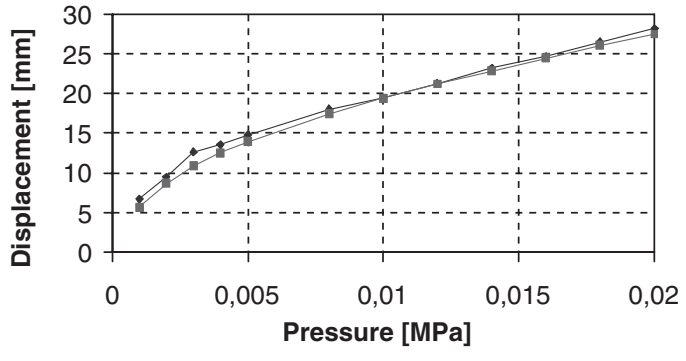

(b) $\rightarrow$ experimental prototype - -numerical model

Figure 8: Comparison between experimental and numerical results in: (a) isometric tests and (b) isotonic tests. 
The isotonic tests were performed until the pressure value of 0.6 bar is reached for five loads: from $9.8 \mathrm{~N}$ to $98 \mathrm{~N}$. The hysteretic behaviour was neglected and the curves were constructed calculating the medium values. In Fig. 9, the results of isometric and isotonic tests are shown.

\subsection{New brace prototype}

A first prototype of the brace is manufactured in order to check the behaviour of the brace assembled with some air pocket devices. The construction of the shell was obtained by a traditional CAM (computer aided manufacturing) system normally used for the base frame of other braces, as Cheneau. The shell was modelled on the trunk of a healthy volunteer, inserting an offset, i.e. a backlash, of about $10 \mathrm{~mm}$ on the upper part. This offset was necessary because of the thickness of the air pocket $(8 \mathrm{~mm})$. A grid of holes was performed on the rear side of the shell, with a correct arrangement to assemble the air pocket devices by valve and nut from the internal side. Two air pocket prototypes were used for this first experimental test. Wearing the brace, made by the shell and the two air pockets, one close to the other, the impression was of too large size, with the devices in a contact-no contact condition with the thorax.

The measurement made by the sensors matrix confirmed this condition. For this reason a covering was necessary to have a uniform thickness in the internal side of the shell. This problem is due to the use of only two air pockets, but it will disappear with the construction of the complete prototype that needs something like 30 air pocket devices. A layer of material, similar to that used for the pad, with a thickness of about $5 \mathrm{~mm}$ was used. It was glued on the internal side of the shell, except where the two air pockets were assembled. After this modification the volunteer had good sensation wearing the brace, with a normal contact between thorax and brace (Fig. 10a). The measurements confirmed this good behaviour of the brace, giving values of the thrust less than $0.5 \mathrm{~N}$. After this step the first experimental tests on the brace started to measure the thrusts due to a single air pocket. The test was carried out in two different position of the air pocket, approximately $3 \mathrm{~B}$ and $4 \mathrm{~B}$, see Fig. $4 \mathrm{~b}$, increasing the pressure value from 0 to $0.065 \mathrm{MPa}$. The measured thrusts, by the sensors matrix, in the two different positions of the device versus the air pressure are very similar, with a maximum value of $25 \mathrm{~N}$ at $0.065 \mathrm{MPa}$. The graph that shows the thrust of one air pocket versus the air pressure is given in Fig. 10b.

The result shows that the maximum value of thrust doesn't meet the design value of $40 \mathrm{~N}$, but it is important that the shape of the graph is not so far from a line. The deficit in the maximum value of the thrust that the device can apply means that a rectangle of four air pockets, $200 \times 200 \mathrm{~mm}^{2}$, can apply a maximum thrust of $100 \mathrm{~N}$, instead of $160 \mathrm{~N}$. That's mean that this new brace could be really interesting considering that the complete brace has a covering of air pockets in the internal side.
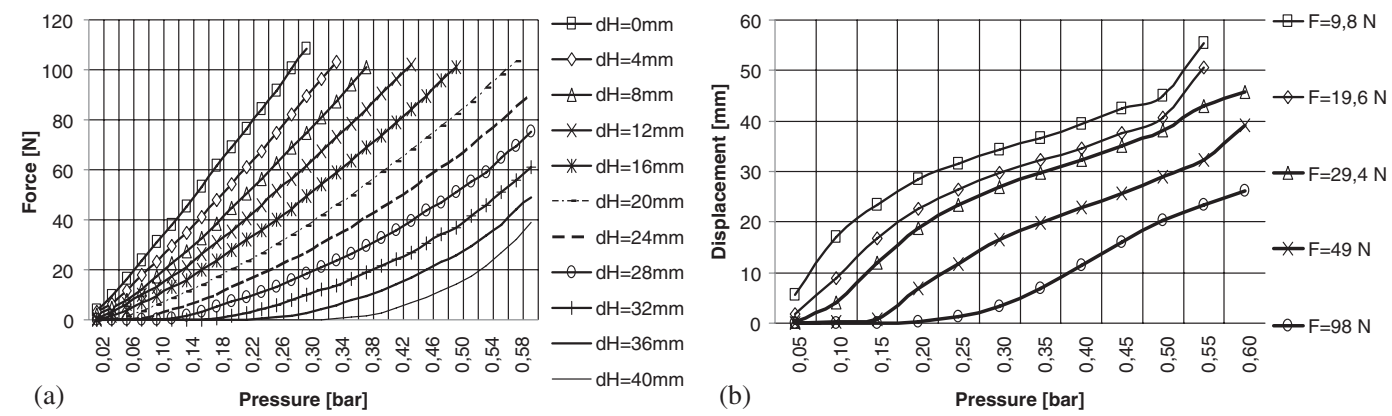

Figure 9: (a) Isometric tests and (b) isotonic tests for the air pocket device. 


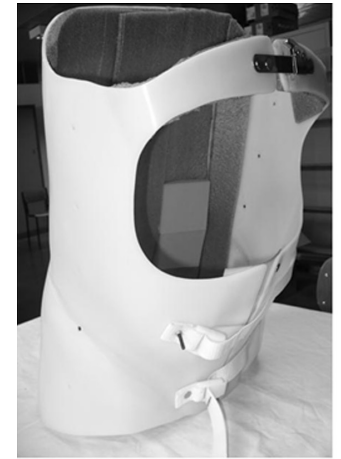

(a)

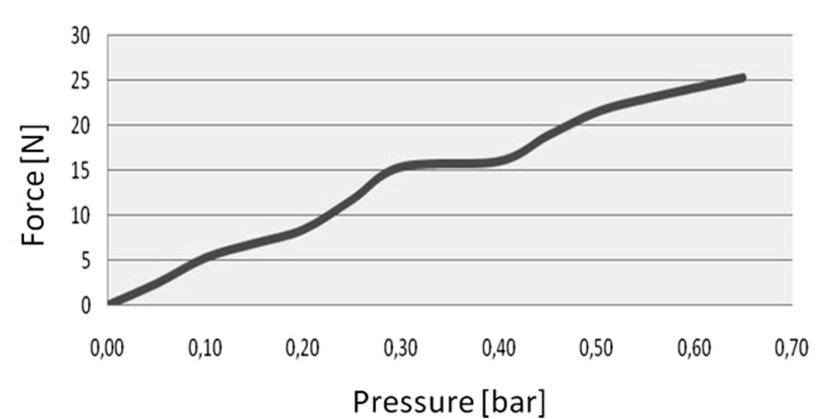

(b)

Figure 10: (a) First prototype of the brace and (b) thrust applied by one air pocket device.

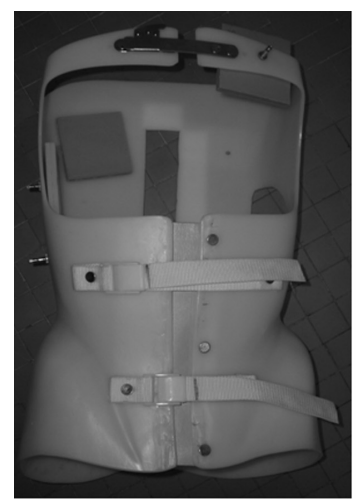

(a)

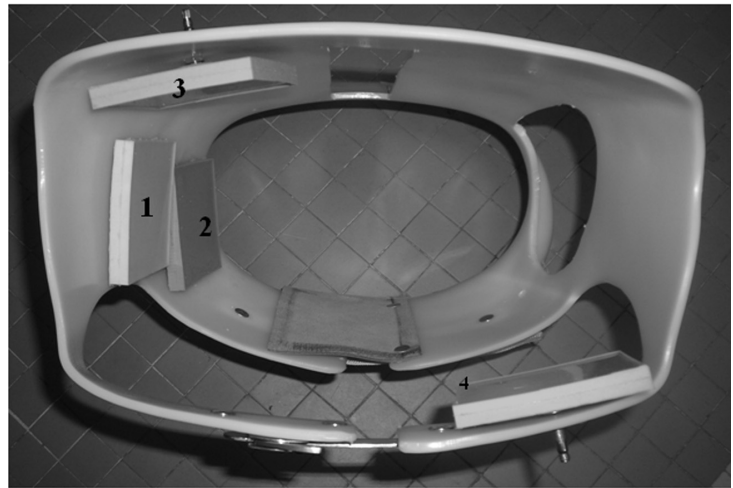

(b)

Figure 11: (a) Frontal view of the second prototype and (b) top view and locations of the air pocket devices.

\section{PRE-CLINICAL EVALUATION OF THE BRACE}

The goal of these experimental tests was to validate the effectiveness of the new brace concept: the threedimensional thrusts system, based on air pocket devices, is able to cause the curvature of the spine? To obtain an answer to these questions the experimental activity was settled to measure the curvature of the spine. The experimental activity was carried out on a female healthy volunteer, aged 26 years and with no history of spine disease, after signing an informed consent form.

A second brace prototype was used. It was modelled on the volunteer and it was manufactured in the same previously described manner. In order to provide an able to be seen spine curvature, thrusts were applied on the spine sector between vertebras T5 and T12. The frame of the brace was modified as follows (Fig. 11). An opening was made in the back part to uncover the vertebras from T6 to L3; a matrix of holes was made on the frame of the brace to allow the air pockets positioning; a triangular shape area of the frame was removed in the left side to allow the rib cage displacements in the opposite part of thrusts application area. Four air pockets were used. The thrusts system was obtained positioning the air pocket devices as shown in Fig. 11b: two air pockets, (1) and (2), in the 
right side of the brace covering the spine sector between vertebras T8 and T12; an air pocket (3) in the back side of the brace, at the level of T6; an air pocket (4) in the front side of the brace, at the level of T5.

To measure the curvature of the spine, a vision system, based on a good quality commercial digital camera with a resolution of 4 megapixels, was used. The photos are captured on the back side of the brace and optical markers are used to measure the spine displacement in both frontal and sagittal plane, as starting idea. The first kind of marker, used to measure displacements in the frontal plane, was made of a black disk with a diameter equal to $12 \mathrm{~mm}$. The disks were directly placed on the spinous processes by glue. The second kind of marker, to be used for displacement measurement in the sagittal plane, was made of a black disk, with a diameter equal to $12 \mathrm{~mm}$, a pin, $30 \mathrm{~mm}$ long, and a second white disk with a diameter equal to $9 \mathrm{~mm}$. One end of the pin was linked to the centre of the first disk; the other was linked to the centre of the second one, so as to be perpendicular to both disks. First disk should be directly placed on the spinous processes by glue. Unfortunately the displacement of the spine caused instability of the second kind of markers, providing false and incongruous displacements of them. For this reason, the measurements were performed only in the frontal plane.

Three load conditions were subsequently applied to the rib cage: the first load condition (FLC) applies thrusts by air pocket 1 and 2 at the same time; the second load condition (SLC) is made of the previous one and of the thrust applied by air pocket 3 ; the third load condition (TLC) is made of the previous one and of the thrust applied by air pocket 4 . The air pressure is the same for all the air pocket devices, $0.05 \mathrm{MPa}$. This pressure is very close to the maximum value, so that the effect on the spine is big and it is easier to detect the displacement.

Markers were placed on the spinous process of vertebras from T6 to L3, for a total amount of 10 markers. For each load condition, besides rest condition (RC), a picture of the back of the brace was captured. The analysis of spine displacements was performed by means of an image analysis of markers positions in the different load conditions. A line was drawn between the centres of two consecutive markers, for a total amount of nine lines, as shown in Fig. 12. In each load condition, inclinations of lines were measured. A comparison among inclinations of each line was performed through load conditions in order to study the evolution of the spine curvature.

Hence, line's inclination, with respect to the vertical direction, at RC, at FLC, at SLC and at TLC were measured and also inclination between RC and FLC, $\delta_{1}$, between FLC and SLC, $\delta_{2}$, between

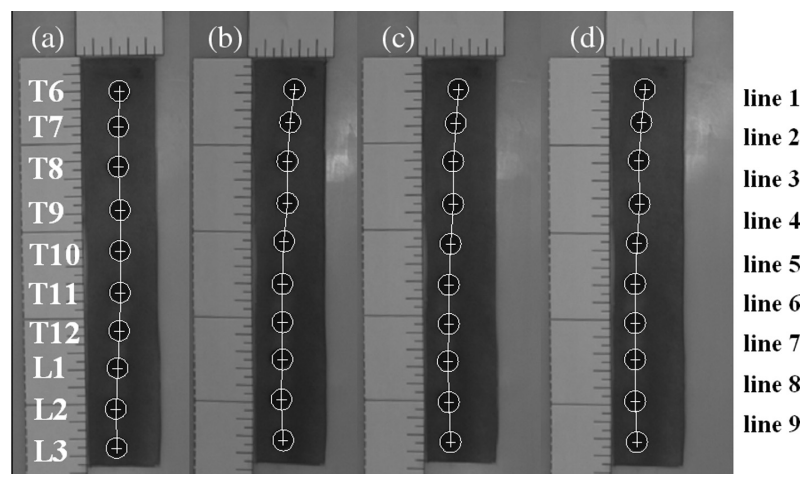

Figure 12: Marker positions at (a) rest condition, (b) first load condition, (c) second load condition, and (d) third load condition. 
SLC and TLC, $\delta_{3}$, and between RC and TLC, $\delta_{\text {tot }}$, as reported in Table 1. Line's inclinations were measured according to the vertical line of the ruler fixed to the brace (Fig. 12). In order to reach the vertical position, positive angle value means a rotation of the line in clockwise direction; negative angle value means a rotation in anticlockwise direction. Positive angle value, between two lines, means a clockwise rotation of the $2^{\text {nd }}$ line with respect to the $1^{\text {st }}$ one; negative angle value means an anticlockwise rotation.

FLC produces a curvature in which the upper sector of the spine (from T11 to T6) has a positive rotation and the lower one (from T12 to L3) a negative rotation. Bigger rotations are registered at the ends of the interested sector of spine. This result is coherent with the thrust system applied. It can be compared to a distributed load on a double fixed beam whose effect is a displacement of the beam in the direction of the load. The same total behaviour is registered in TLC. The effects of SLC and TLC are less evident, probably because their actions involve displacements in the sagittal plane. Nevertheless, the almost absence of rotations in the sector between vertebras T7 and L1 (from line 2 until line 7) suggests a torsion of the spine in the upper zone. In fact, the only significant rotations are registered in correspondence of line 1, both in SLC and in TLC, at the level of which thrusts were applied. FLC provided a maximum displacement of the spine, towards the left zone of the back window, equal to $5 \mathrm{~mm}$; SLC equal to $7.5 \mathrm{~mm}$; TLC equal to $9 \mathrm{~mm}$. Taking into account the congruent correlation between thrusts and spine displacements, it follows that the applied thrusts system is able to produce a displacement of the spine.

\section{CONCLUSIONS}

This paper deals about an innovative brace for scoliosis treatment. The idea is to apply the thrust in more than three points, as is usual in many braces. The brace here proposed is based on the 'global thrust', that is a three-dimensional thrusts system acting on the entire rib cage at many levels of the spine.

The frame of the new brace is made with plastic shell and an internally fixed covering of air pocket devices. Each air pocket is made of silicon rubber and the internal air pressure can be regulated and checked. Starting from the measurement of typical thrust values in commercial braces, air pocket devices are designed and then manufactured. In order to optimise the air pocket device design and because of the constitutive law of the silicon rubber, a numerical model, implemented by a finite element code, is developed and then validated. Air pocket device is subjected to several tests to be

Table 1: Lines' inclination measured in the frontal plane.

\begin{tabular}{|c|c|c|c|c|c|c|c|c|}
\hline Line no. & $\begin{array}{c}\text { Slope at } \\
\text { RC }\end{array}$ & $\begin{array}{l}\text { Slope at } \\
\text { FLC }\end{array}$ & $\begin{array}{c}\text { Slope at } \\
\text { SLC }\end{array}$ & $\begin{array}{c}\text { Slope at } \\
\text { TLC }\end{array}$ & $\delta_{1}$ & $\delta_{2}$ & $\delta_{3}$ & $\delta_{\text {tot }}$ \\
\hline 1 & $+1^{\circ}$ & $+8^{\circ}$ & $+4^{\circ}$ & $+6^{\circ}$ & $+7^{\circ}$ & $-4^{\circ}$ & $+2^{\circ}$ & $+5^{\circ}$ \\
\hline 2 & $0^{\circ}$ & $+4^{\circ}$ & $+4^{\circ}$ & $+4^{\circ}$ & $+4^{\circ}$ & $0^{\circ}$ & $0^{\circ}$ & $+4^{\circ}$ \\
\hline 3 & $-2^{\circ}$ & $0^{\circ}$ & $0^{\circ}$ & $-1^{\circ}$ & $+2^{\circ}$ & $0^{\circ}$ & $-1^{\circ}$ & $+1^{\circ}$ \\
\hline 4 & $0^{\circ}$ & $+5^{\circ}$ & $+4^{\circ}$ & $+4^{\circ}$ & $+5^{\circ}$ & $-1^{\circ}$ & $0^{\circ}$ & $+4^{\circ}$ \\
\hline 5 & $0^{\circ}$ & $+2^{\circ}$ & $+2^{\circ}$ & $+2^{\circ}$ & $+2^{\circ}$ & $0^{\circ}$ & $0^{\circ}$ & $+2^{\circ}$ \\
\hline 6 & $+1^{\circ}$ & $0^{\circ}$ & $0^{\circ}$ & $0^{\circ}$ & $-1^{\circ}$ & $0^{\circ}$ & $0^{\circ}$ & $-1^{\circ}$ \\
\hline 7 & $+3^{\circ}$ & $0^{\circ}$ & $+1^{\circ}$ & $0^{\circ}$ & $-3^{\circ}$ & $+1^{\circ}$ & $-1^{\circ}$ & $-3^{\circ}$ \\
\hline 8 & $+2^{\circ}$ & $+1^{\circ}$ & $-1^{\circ}$ & $0^{\circ}$ & $-1^{\circ}$ & $-2^{\circ}$ & $+1^{\circ}$ & $-2^{\circ}$ \\
\hline 9 & $+1^{\circ}$ & $-2^{\circ}$ & $-2^{\circ}$ & $-2^{\circ}$ & $-3^{\circ}$ & $0^{\circ}$ & $0^{\circ}$ & $-3^{\circ}$ \\
\hline
\end{tabular}


characterised in terms of maximum output force, maximum operative air pressure, air leaks, and maximum burst pressure. Finally, isometric and isotonic tests are performed to define a control chart of air pocket devices based on the relationship of pressure, force and displacement values. The final experimental activity, on a healthy volunteer, shows that a thrusts system made by air pocket devices is able to obtain spine displacements. The encouraging results say that the new brace concept can be an alternative to the actual scoliosis braces. The work is in progress and the next step will be a clinical validation on ill subjects by a complete prototype.

\section{ACKNOWLEDGEMENTS}

The authors are thankful to Miss Silvia Sparvieri, as well as to Ortopedia Picena of Guiducci Quirino, Ascoli Piceno, Italy, for their kind support. A special thanks to Novatec Spinoff s.r.l. for its support to this research.

\section{REFERENCES}

[1] Negrini, S., Aulisa, L., Ferraro, C., Fraschini, P., Masiero, S., Simonazzi, P., Tedeschi, C. \& Venturin, A., Italian guidelines on rehabilitation treatment of adolescents with scoliosis or other spinal deformities. Eura Medicophys, 41, pp. 183-201, 2005.

[2] Shangguan, L., Fan, X. \& Ming, L.I., Inherence involved in the pathogenesis of idiopathic scoliosis. EXCLI Journal, 7, pp. 104-114, 2008.

[3] Guille, J.T., D'Andrea, L.P. \& Betz, R.R., Fusionless treatment of scoliosis. Orthopedic Clinics of North America, 38, pp. 541-545, 2007. doi:10.1016/j.ocl.2007.07.003

[4] Labelle, H., Dansereau, J., Bellefleur, C. \& Poitras, B., Three-dimensional effect of the Boston brace on the thoracic spine and rib cage. Spine, 21(1), pp. 59-64, 1996. doi:10.1097/00007632199601010-00013

[5] Bignardi, C., Bondente, P.G. \& Rosso, V., Non invasive measurement of body jacket pressures in scoliosis. XIV International INTERBOR World Congress, Boston, USA, July 14-17th, p. 31, 1999.

[6] ANSYS Theoretical Manual, Swanson Analysis System Inc.: Houston, 1989.

[7] Ferry, J.D., Viscoelastic Properties of Polymers, John Wiley \& Sons, Inc.: New York, 1980. 